Pacific Journal of Mathematics

A NOTE ON AUTOMORPHISMS OF LIE ALGEBRAS 


\section{A NOTE ON AUTOMORPHISMS OF LIE ALGEBRAS}

\section{N. JACOBSON}

In a beautiful paper which appeared in 1939 ([4]), F. Gantmacher made a thorough study of automorphisms of semi-simple Lie algebras over the field of complex numbers. Among other things, he defined the index $n\left(G_{i}\right)$ of a connected component $G_{i}$ of the automorphism group $G=G(2)$ as the minimum multiplicity of the characteristic root 1 for elements of $G_{i}$. The main purpose of this note is the determination of these indices. It is somewhat surprising that this does not appear in Gantmacher's paper since all the methods for deriving the formula for index $G_{i}$ are available in his paper. The secondary purpose of this note is to extend Gantmacher's theory to the case of Lie algebras over arbitrary algebraically closed base fields of characteristic 0 . This can be done by using algebraic group concepts and techiques which are by now well known. Nevertheless, it seems worthwhile to carry out the program in detail since Gantmacher's results give a real insight into the action of an automorphism in a semi-simple Lie algebra. For example, as we indicate, they can be used to give a new derivation and sharpening of theorems on fixed points which are due to Borel and Mostow ([1]).

1. Generalities on automorphisms. Let $\stackrel{?}{\sim}$ be a finite dimensional Lie algebra over an algebraically closed field $\Phi$ of characteristic $0, G$ the group of automorphisms of $2 . G$ is an algebraic group and it has a decomposition $G=G_{0} \cup G_{1} \cup \cdots \cup G_{r-1}$ where $G_{i}$ are the algebraic components and $G_{0}$ is the component of the identity element 1 . This is an invariant subgroup of finite index $r$ in $G$ and is irreducible, which means that the intersection of any two nonvacuous open subsets in $G_{0}$ is nonvacuous. The topology referred to here is the Zariski topology in which open sets are the complements of algebraic subsets of $G$.

If $\eta$ is a nonsingular linear transformation in $2, \eta$ has a unique decomposition as $\eta=\eta_{s} \eta_{u}=\eta_{u} \eta_{s}$ where $\eta_{s}$ and $\eta_{u}$ are polynomials in $\eta$, $\eta_{s}$ is semi-simple and $\eta_{u}$ is unipotent, that is, $\eta_{u}-1$ is nilpotent. If $\eta$ is an automorphism then $\eta_{u}$ and $\eta_{s}$ are automorphisms. If $\eta$ is a unipotent automorphism then

$$
\log \eta=(\eta-1)-\frac{1}{2}(\eta-1)^{2}+\frac{1}{3}(\eta-1)^{3}-\cdots
$$

Received February 27, 1961. This research was supported in part by the United States Air Force through the Air Force Office of Scientific Research and Development Command under Contract No. AF 49 (C38) 515. Reproduction in whole or in part is permitted for any purpose of the United States Government. 
is a nilpotent derivation. Conversely, if $D$ is a nilpotent derivation then $\eta=\exp D$ is a unipotent automorphism. Any unipotent automorphism is contained in the algebraic component of 1 . Hence an automorphism $\eta$ and its semi-simple part $\eta_{s}$ are contained in the same component.

If $\eta$ is a linear transformation in $\mathscr{L}$ we can decompose $\mathfrak{L}=\mathfrak{L}_{\alpha} \oplus$ $\mathfrak{R}_{\beta} \oplus \cdots \oplus \mathfrak{R}_{\rho}$ where $\mathfrak{R}_{\alpha}$ is the characteristic space of $\eta$ corresponding to the characteristic root $\alpha: \Omega_{\alpha}=\left\{x_{\alpha} \mid x_{\alpha}(\eta-\alpha 1)^{k}=0\right.$ for some $\left.k\right\}$. The semi-simple part $\eta_{s}$ is the linear transformation which leaves each $\Omega_{a}$ invariant and coincideds with the scalar multiplication $\alpha 1$ in $\mathfrak{R}_{\alpha}$. If $\eta$ is an automorphism then $\left[\mathfrak{R}_{\alpha} \mathfrak{R}_{\beta}\right]=0$ if $\alpha \beta$ is not a characteristic root and $\left[\mathfrak{R}_{\alpha} \mathfrak{R}_{\beta}\right] \subseteq \mathfrak{R}_{\alpha \beta}$ if $\alpha \beta$ is a characteristic root. Here $\left[\mathbb{R}_{\alpha} \mathbb{R}_{\beta}\right]$ is the subspace spanned by the Lie products $\left[x_{\alpha} x_{\beta}\right], x_{\alpha} \in \mathfrak{R}_{\alpha}, x_{\beta} \in \mathfrak{R}_{\beta}$. In particular, $\mathfrak{R}_{1}$ the space of the characteristic root 1 is a subalgebra and $\left[\mathbb{R}_{\alpha} \mathbb{R}_{1}\right] \subseteq \mathbb{R}_{\alpha}$. In most considerations the refined decomposition $\mathbb{L}=\Sigma \mathbb{R}_{\alpha}$ will be replaced by a coarser Fitting type decomposition: $\mathbb{R}=\mathfrak{R}_{1} \oplus \mathfrak{R}$ where $\mathfrak{R}=\sum_{\alpha \neq 1} \mathfrak{R}_{\alpha}$. These two spaces are invariant under $\eta, \eta$ is unipotent in $\mathscr{L}_{1}$ and $\eta-1$ is nonsingular in $\mathfrak{R}$. We have $\left[\mathfrak{R} \Omega_{1}\right] \subseteq \mathfrak{R}$. If $\eta$ is semi-simple then $\mathfrak{L}_{1}$ is the set of fixed points under $\eta$ and $\mathfrak{R}=\mathbb{2}^{\eta-1}=\left\{x^{\eta}-x \mid x \in \mathbb{L}\right\}$.

As before, let $G=G_{0} \cup G_{1} \cup \cdots \cup G_{r-1}$ be the decomposition of $G$ into its algebraic irreducible components. We define the index $n\left(G_{i}\right)$ of $G_{i}$ as the minimum multiplicity of the characteristic root 1 for the $\eta \in G_{i}$. The multiplicity of the root is the same as the dimensionality of the corresponding characteristic space. An element $\eta \in G_{i}$ is called regular if $\operatorname{dim} \mathfrak{Q}_{1}(\eta)=\operatorname{index} G_{i}$. Let $\left(u_{1}, \cdots, u_{n}\right)$ be a basis for $\mathfrak{\&}$ and let $(\alpha)$ be a matrix of $\eta$ relative to this basis. Write the characteristic polynomial of $(\alpha)-1$ as

$$
f_{\eta}(\lambda)=\lambda^{n}-\rho_{1}(\eta) \lambda^{n-1}+\cdots+(-1)^{n} \rho_{n}(\eta) .
$$

The mappings $\eta \rightarrow \rho_{j}(\eta)$ are polynomial functions. If the index of $G_{i}=$ $l_{i}$, then $\rho_{n}(\eta)=\cdots=\rho_{n-l_{i}+1}(\eta)=0$ for all $\eta \in G_{i}$ but $\rho_{n-l_{i}}(\eta) \not \equiv 0$ on $G_{i}$. The regular elements of $G_{i}$ are those such that $\rho_{n-l_{i}}(\eta) \neq 0$. Hence they form an open set in $G_{i}$.

2. Lie Algebras of algebraic groups. We need to recall some notions on linear algebraic groups. The results we shall quote can be found in two books by Chevalley ([3]). We recall first that if $V$ and $W$ are finite dimensional vector spaces, a rational mapping $R$ of $V$ into $W$ is a mapping of the form $x=\Sigma \xi_{i} e_{i} \rightarrow y=\Sigma \eta_{j} f_{j}$ where $\eta_{j}=R_{j}(\xi) \equiv R_{j}\left(\xi_{1}, \cdots, \xi_{m}\right)$ are rational functions of the $\xi$ 's. Here $\left(e_{1}, \cdots, e_{m}\right)$ is a basis for $V$ and $\left(f_{1}, \cdots, f_{n}\right)$ is a basis for $W$. The rational mapping is defined on an open subset of $V$. An important special case is that in which $W=\Phi$. Then $R$ is a rational function on $V$. In the general case, if $a$ is a point at which $R$ is defined, the differential $D_{a}$ of $R$ at $a$ is the linear map- 
ping $x \rightarrow\left(D_{a} R\right)(x)$ where

$$
\left(D_{a} R\right)(x)=\sum_{1}^{n}\left(\sum_{1}^{m}\left(\frac{\partial R_{j}}{\partial \lambda_{k}}\right)_{\lambda_{h}=x_{h}} \xi_{k}\right) f_{j}
$$

for $a=\Sigma \alpha_{i} e_{\imath}$. Here $\partial R_{j} / \partial \lambda_{k}$ is the formal partial derivative of the rational expression $R_{j}\left(\lambda_{1}, \cdots, \lambda_{m}\right)$ in indeterminates $\lambda$ with respect to the indeterminate $\lambda_{k}$. Let $E$ be an irreducible set in $V$, that is, the ideal $\mathfrak{U}$ of polynomials $P\left(\lambda_{1}, \cdots, \lambda_{m}\right)$ which are 0 at every point of $E$ is prime. Let $a$ be a point of $E$. We define the tangent space to $E$ at $a$ to be the subspace of vectors $x$ in $V$ such that $\left(D_{a} P\right)(x)=0$ for every $P$ in the ideal $\mathfrak{A}$ corresponding to $E$. The condition on $x=\Sigma \xi_{k} e_{k}$ are: $\sum_{k}\left(\partial P / \partial \lambda_{k}\right)_{\lambda_{h}=\alpha_{h}} \xi_{k}=0$ for all $P \in \mathfrak{A}$. It suffices to have these conditions satisfied for a set of generators $\left(P_{1}, \cdots, P_{q}\right)$ of $\mathfrak{A}$. If $R$ is a rational mapping defined on $E$ then $R(E)$ is irreducible and $D_{a} R$ maps the tangent space to $E$ at $a \in E$ into a subspace of the tangent space at $R(a)$ of $R(E)$.

If $G$ is an irreducible algebraic linear group the tangent space \& at 1 for $G$ can be made a Lie algebra by identifying its elements with derivations in the algebra of polynomial functions on $G$. The dimensionality of $\mathcal{Q}$ is the same as that of $G$. If $\mathcal{Q}$ is any Lie algebra and $G_{0}$ is the component of 1 of the group of automorphisms then the Lie algebra of $G_{0}$ is the Lie algebra of derivations of $\mathbb{R}$. If $\mathcal{Z}$ is semi-simple then all the derivations are inner and the Lie algebra of $G_{0}$ is $a d \&$ the set of adjoint mappings ad $a: x \rightarrow[x a]$ in $\mathfrak{Z}$.

3. Regular automorphisms of semi-simple Lie algebras. We shall now derive the purely algebraic form of Gantmacher's results on automorphisms of semi-simple Lie algebras.

Let $\&$ be semi-simple and let $\eta$ be an automorphism of $\mathbb{R}, \mathbb{Z}=\mathbb{R}_{1} \oplus$ $\mathfrak{R}$ the Fitting decomposition of $\mathbb{Q}$ relative to $\eta$ Thus $\mathfrak{R}_{1}$ is the space of the characteristic root $1, \mathfrak{N}$ the sum of the other characteristic spaces, $\mathfrak{Q}_{1}$ is a subalgebra and $\left[\mathfrak{R} \mathfrak{\Omega}_{1}\right] \subseteq \mathfrak{R}$. We note first

\section{THEOREM 1. $\quad \mathfrak{Z}_{1}$ is a reductive subalgebra of $\mathbb{2}$.}

Proof. The assertion is that the subalgebra $a d_{\Omega} Q_{1}$ of the Lie algebra

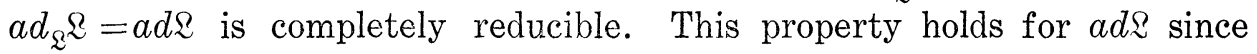
$\mathcal{Q}$ is semi-simple. If $\eta_{s}$ is the semi-simple part of $\eta$ then $\mathscr{Q}_{1}=\left\{l_{1} \mid l_{1}^{n_{s}}=l_{1}\right\}$. This is equivalent to $\left[\eta_{s}, a d l_{1}\right]=0$ and this implies that $a d_{\Omega_{2}} \mathfrak{Q}_{1}$ is a splittable Lie algebra of linear transformations in the sense of Malcev. Also

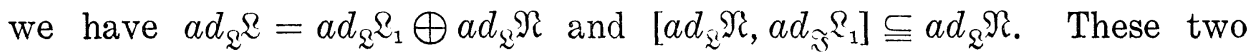
properties imply that $a d_{\Omega} Q_{1}$ is completely reducible ([6] p. 109).

Since $\mathfrak{L}_{1}$ is reductive we have $\mathfrak{L}_{1}=\mathfrak{L}_{1}^{\prime} \oplus \widetilde{C}$ where $\mathfrak{L}_{1}^{\prime}$ is the derived 
algebra of $\Omega_{1}$ and is semi-simple; $\mathfrak{C}$ is the center of $\mathfrak{L}_{1}$ and $a d_{\Sigma_{2}} c$ is semisimple for every $c$ in $c([6]$ p. 106).

THEOREM 2. If $\eta$ is regular $\mathfrak{L}_{1}$ is abelian and $\eta$ is semi-simple.

Proof. Suppose $\mathfrak{Z}_{1}$ is not abelian so that $\mathfrak{R}_{1}^{\prime}$ is a nonzero semi-simple Lie algebra. There exist elements $z$ and $w$ in $\mathfrak{R}_{1}^{\prime}$ such that $a d_{\mathfrak{R}_{1}^{\prime}}$ and $a d_{\mathfrak{Q}_{1}^{\prime}} w$ are nilpotent derivations and $\left(\exp a d_{\mathfrak{Z}_{1}^{\prime}} z\right)\left(\exp a d_{\mathfrak{Z}_{1}^{\prime}} w\right)$ is an automorphism of $\mathfrak{Q}_{1}^{\prime}$ which is not unipotent. ${ }^{1)}$ Since $a d_{\mathcal{R}_{1}^{\prime}} z$ and $a d_{\mathcal{I}_{1}^{\prime}} w$ are nilpotent it is known that $a d z \equiv a d_{\Omega} z$ and $a d w$ are nilpotent. If $\alpha, \beta \in \Phi$ we can form the automorphism $(\exp \alpha a d z)(\exp \beta a d w)$ which is in the com-

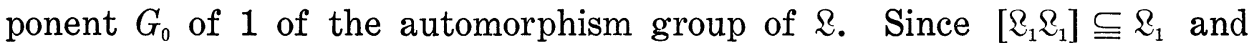
$\left[\mathfrak{R R _ { 1 }}\right] \subseteq \mathfrak{R}$ it follows that the automorphism

$$
\zeta(\alpha, \beta)=\eta_{s}(\exp \alpha \operatorname{ad} z)(\exp \beta \operatorname{ad} w)
$$

satisfies $\mathfrak{R}_{1}^{\zeta(\alpha, \beta)} \subseteq \mathfrak{R}_{1}, \mathfrak{N}^{\zeta(\alpha, \beta)} \subseteq \mathfrak{R}$. Since $\eta_{s}=1$ in $\mathfrak{R}_{1}$ the restriction of $\zeta(1,1)$ to $\Omega_{1}$ is not unipotent. Also the restriction of $\zeta(0,0)-1=\eta_{s}-$ 1 to $\Re$ is nonsingular. It follows by a standard argument that $\alpha, \beta$ can be chosen so that the multiplicity of the characteristic root 1 for $\zeta(\alpha, \beta)$ is less than $\operatorname{dim} \Omega_{1}$. Since $\zeta(\alpha, \beta)$ is in the same component as $\eta$ this means that $\eta$ is not regular contrary to assumption. Hence $\mathfrak{R}_{1}$ is abelian. Then every $a d_{\Omega} l_{1}, l_{1} \in \Omega_{1}$ is semi-simple. On the other hand, $\log \eta_{u}$ is a nilpotent derivation in $\mathfrak{L}$. Since the derivations of $\mathbb{L}$ are all inner, $\log \eta_{u}=a d z, z \in \mathfrak{R}$. Since $\left[\eta, \log \eta_{u}\right]=0, z \in \mathfrak{R}_{1}$. Hence $a d z$ is nilpotent and semi-simple. Then $a d z=0$ which implies that $\eta_{u}=1$ and $\eta=\eta_{s}$ is semi-simple.

We wish to prove the converse of Theorem 2. For this we shall need the following

LEMma. Let $\eta$ be an automorphism of a semi-simple Lie algebra \& such that the subalgebra $\mathfrak{Q}_{1}$ is abelian. Let $H$ be the subgroup of the automorphisms group $G$ of $\&$ of elements commuting with $\eta, H_{0}$ the algebraic component of 1 of $H, G_{0}$ the algebraic component of 1 of $G$. Then

$$
K=\left\{\tau \eta \zeta \tau^{-1} \mid \tau \in G_{0}, \zeta \in H_{0}\right\}
$$

contains a nonvacuous open subset of $\eta G_{0}$.

Proof. We note first that the proof of Theorem 2 shows that every $\zeta \in H_{0}$ is semi-simple; hence every $\eta^{\prime}=\eta \zeta=\zeta \eta$ is semi-simple. The Lie algebra of $G_{0}$ is $a d \&$ and the Lie algebra of $H_{0}$ is $a d_{\Omega} \Omega_{1}$ since $\&_{1}$ is the

1 For example, we can take $z, w$ to satisfy $[z w]=h \neq 0,[z h]=2 z,[w h]=-2 w$. See the proof of the lemma to Th. 5 . 
set of fixed points under $\gamma_{\text {. }}$. If $\xi, \zeta \in H_{0}$ then the mapping $\xi \rightarrow \zeta^{-1} \xi \zeta$ is the identity; hence the induced mapping $x \rightarrow \zeta^{-1} \times \zeta$ is the identity in $a d_{\Omega} \Omega_{1}$. Since $\zeta^{-1}\left(a d l_{1}\right) \zeta=a d l_{1}^{\zeta}$ it follows that $l_{1}^{\zeta}=l_{1}$. This implies that the space of fixed points $\Omega_{1}\left(\eta^{\prime}\right) \supseteqq \Omega_{1}(\eta)$ for any $\eta^{\prime}=\eta \zeta$. We note next that $K$ is the orbit of $\eta H_{0}$ under the group of mappings $\xi \rightarrow \tau \xi \tau^{-1}, \tau \in G_{0}$. This implies that $K$ is épais in the sense of Chevalley: $K$ is irreducible and $K$ contains a nonvacuous open subset of its closure (see Chevalley [3] tome III, p. 193). The result we wish to prove will now follow by showing that $K$ is dense in $\gamma_{1} G_{0}$. (This is all which will be needed for the proof of Theorem 3.) Let $\eta^{\prime}=\eta \zeta$ be any element of $\eta H_{0}$. Then the tangent space $\mathfrak{I}\left(K ; \eta^{\prime}\right)$ of $K$ at $\eta^{\prime}$ contains the tangent space $\mathfrak{I}\left(\eta_{3} ; \gamma^{\prime}\right)$ of $\eta H_{0}$ at $\eta^{\prime}$ as well as the image of $\eta^{\prime}$ under the Lie algebra of mappings $\mathrm{x} \rightarrow[\mathrm{x}, a d l], l \in L$ (Chevalley, loc. cit. p. 192). This is the set of mappings $\left[\gamma^{\prime}, a d l\right], l \in \mathfrak{R}$. Now $\mathfrak{I}\left(H_{0} ; 1\right)$ is the Lie algebra $a d_{\Omega^{2}}{ }_{1}$ so $\mathfrak{I}\left(H_{0}, \eta^{\prime}\right)=\mathfrak{I}\left(\eta^{\prime} H_{0} ; \eta^{\prime}\right)=\eta^{\prime} a d_{\Omega^{2}}$. We wish to show that $\mathfrak{I}\left(K ; \eta^{\prime}\right)=$ $\mathfrak{I}\left(\gamma G_{0} ; \gamma^{\prime}\right)=\eta^{\prime}$ ad?. Since $\mathfrak{I}\left(K, \eta^{\prime}\right)$ contains $\eta^{\prime} a d_{\Sigma^{2}} \mathbb{R}_{1}$ and $\left[\eta^{\prime}, a d \Omega\right]=$ $\left\{\left[\eta^{\prime}, a d l\right], l \in \Omega\right\}$, it is enough to show that ad $\mathbb{Z}=a d_{Z^{2}}{ }_{1}+\left(\eta^{\prime}\right)^{-1}\left[\eta^{\prime}\right.$, ad $\left.:\right]$. Since $\left(\eta^{\prime}\right)^{-1}\left[\eta^{\prime}, a d l\right]=a d l-a d l^{\eta^{\prime}}=a d\left(l-l^{\eta^{\prime}}\right)$, it suffices to show that

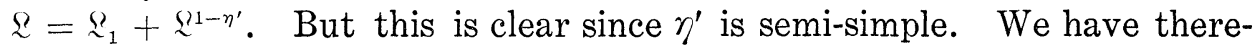
fore proved that the tangent spaces to $K$ and to $\gamma G_{0}$ at any point $\eta^{\prime}=\eta \xi$ coincide. Since $K$ is the orbit of $\eta H_{0}$ under the set of mappings $\xi \rightarrow \tau \xi \tau^{-1}$ it follows that the tangent space to $K$ and to $\eta G_{0}$ at any point of $K$ coincide. This implies that $K$ is dense in $\gamma G_{0}$.

\section{Theorem 3. If $\mathfrak{L}_{1}$ is abelian then $\eta$ is regular.}

Proof. Let $K$ be the set defined by (2). Then the lemma implies that $K$ contains a regular element $\tau \eta \zeta \tau^{-1}, \zeta \in H_{0}, \tau \in G_{0}$. Then $\gamma \zeta$ is a regular element contained in the component $G_{\imath}=\gamma_{i} G_{0}$ containing $\eta$. The foregoing proof shows also that $\Omega_{1}(\gamma, \zeta) \supseteqq \mathfrak{R}_{1}\left(\gamma_{)}\right)$. Since $\eta \zeta$ is regular we have $\mathfrak{Z}_{1}(\eta \zeta)=\mathfrak{Z}_{1}(\eta)$ so $\eta$ is regular also.

Let $\eta$ be a regular element and, as before, let $H_{0}(\eta)$ be the algebraic component of 1 in the subgroup $H(\eta)$ of $G$ of elements commuting with $\eta$. Then $H_{0}(\eta) \eta=\eta H_{0}(\eta)$ is the component of $\eta$ in $H(\eta)$. As we have seen in the preceding proof, the Lie algebra $H_{0}(\eta)$ is $\operatorname{ad}_{i=1}(\eta)$ where $\Omega_{1}(\eta)$ is the set of fixed points under $\eta$. Moreover, $\Omega_{1}(\eta)$ and $H_{0}(\eta)$ are abelian. The fact that $H_{0}(\eta)$ is abelian implies that $H_{0}(\eta) \subseteq H_{0}\left(\eta^{\zeta}\right)$ for any $\zeta \in H_{0}(\eta)$. If $\eta \zeta$ is regular also then we have $H_{0}(\eta \zeta)=H_{0}(\eta)$. In a similar manner, we see that the elements of $\Omega_{1}(\eta)$ are fixed under $\eta \zeta$. If $\eta \zeta$ is regular also then $\xi_{1}(\eta)=?_{1}(\eta \zeta), \zeta \in H_{0}(\eta)$. We note also that the argument used in the proof of Theorem 2 shows that every element of $H_{0}(\eta)$ and hence of $H_{0}(\eta) \eta$ is semi-simple.

We now use the full force of the lemma to prove the following 
THEOREM 4. Let $\eta_{1}$ and $\eta_{2}$ be two regular automorphisms contained in the same component of the automorphism group. Let $\mathfrak{L}_{1}\left(\eta_{j}\right)$ be the subalgebra of $\mathbb{2}$ of fixed elements under $\eta_{j}, j=1,2$, and let $H_{0}\left(\eta_{j}\right)$ be the algebraic component of 1 in the subgroup $H\left(\eta_{j}\right)$ of automorphisms commuting with $\eta_{j}$. Then there exists a $\tau \in G_{0}$ such that $\Omega_{1}\left(\eta_{1}\right)^{\tau}=\Omega_{1}\left(\eta_{2}\right)$ and $\eta_{2} H_{0}\left(\eta_{2}\right)=\tau^{-1}\left(\eta_{1} H_{0}\left(\eta_{1}\right)\right) \tau$.

Proof. Let $K_{1}$ and $K_{2}$ be the sets (2) defined by $\eta_{1}$ and $\eta_{2}$ respectively. Then $K_{1}$ and $K_{2}$ contain open subsets of the component $G_{i}$ containing $\eta_{1}$ and $\eta_{2}$. It follows that there exists a regular element in $K_{1} \cap K_{2}$. Hence there exist $\tau_{j} \in G_{0}, \zeta_{j} \in H_{0}\left(\eta_{j}\right)$ such that

$$
\eta=\tau_{1}\left(\eta_{1} \zeta_{1}\right) \tau_{1}^{-1}=\tau_{2}\left(\eta_{2} \zeta_{2}\right) \tau_{2}^{-1}
$$

is regular. Then $\eta_{1} \zeta_{1}$ and $\eta_{2} \zeta_{2}$ are regular elements of $G_{i}$ and $\eta_{2} \zeta_{2}=$ $\tau^{-1}\left(\eta_{1} \zeta_{1}\right) \tau$ where $\tau=\tau_{1}^{-1} \tau_{2}$. Then $\mathfrak{I}_{1}\left(\eta_{2}\right)=\mathfrak{R}_{1}\left(\eta_{2} \zeta_{2}\right)=\mathfrak{R}_{1}\left(\eta_{1} \zeta_{1}\right)^{\tau}=\mathfrak{R}_{1}\left(\eta_{1}\right)^{\tau}$. In a similar manner we see that $\tau^{-1}\left(\eta_{1} H_{0}\left(\eta_{1}\right)\right) \tau=\eta_{2} H_{0}\left(\eta_{2}\right)$.

We have noted that if $\eta$ is regular every element of $\eta H_{0}(\eta)$ is semisimple. We wish to prove that conversely any semi-simple automorphism belongs to an $\eta H_{0}(\eta)$ where $\eta$ is regular. The proof of this result in the complex case given by Gantmacher is based on the use of exponentials of elements $a d l_{1}, l_{1} \in \mathfrak{R}_{1}$. These are not available in the algebraic case. However, a suitable substitute for these has been found by Seligman and we shall use these.

Let $\Re$ be a semi-simple subalgebra of a Lie algebra $\mathbb{Q}, \mathfrak{S}$ a Cartan subalgebra of $\Omega$. Let $\alpha_{i}, i=1,2, \cdots, l$ be a simple system of roots for $\Omega$ relative to $\mathfrak{g}, \Omega_{\alpha_{i}}$ the corresponding one dimensional root spaces. Then there exists a canonical set of generators $e_{i}, f_{i}, h_{i}, i=1,2, \cdots, l$, for $\Omega$ such that the $h_{i}$ form a basis for $\mathfrak{g}, e_{i} \in \Re_{\alpha_{i}}, f_{i} \in \Re_{-\alpha_{i}}$ and the following relations hold:

$$
\begin{aligned}
{\left[h_{i} h_{j}\right] } & =0 \\
{\left[e_{i} f_{j}\right] } & =\delta_{i j} h_{i} \\
{\left[e_{i} h_{j}\right] } & =A_{j i} e_{i} \\
{\left[f_{i} h_{j}\right] } & =-A_{j i} f_{i}
\end{aligned}
$$

where $\left(A_{i j}\right)$ is the Cartan matrix of the simple system $\alpha_{i}$. We have $A_{i i}=2$ and $A_{i j}$ is a nonpositive integer if $i \neq j$. It is known that the mappings $a d e_{i}$ and $a d f_{i}$ are nilpotent (in $\mathfrak{2}$ ). Following Seligman we introduce the automorphism in 2 :

$$
\sigma_{i}(\xi)=\left(\exp a d \xi e_{i}\right)\left(\exp a d \xi^{-1} f_{i}\right)\left(\exp a d \xi e_{i}\right)
$$

where $\xi$ is any nonzero element of $\Phi$ and $i=1,2, \cdots, l$. Also we set 


$$
\omega_{i}(\xi)=\sigma_{i}(\xi) \sigma_{i}(-1)
$$

and we let $H$ be the group of automorphisms of $\mathbb{Q}$ generated by the $\omega_{i}(\xi)$, $\xi \neq 0$ in $\Phi, i=1,2, \cdots, l$. Clearly the $\omega_{i}(\xi)$ map $\Re$ into itself. It has been shown by Seligman that the restriction $\widetilde{H}$ of $H$ to $\Re$ coincides with the group of automorphism in $\Re$ such that

$$
e_{i} \rightarrow \mu_{i} e_{i}, f_{i} \rightarrow \mu_{i}^{-1} f_{i}, h_{i} \rightarrow h_{i}
$$

where the $\mu_{i}$ are arbitrary nonzero elements. ${ }^{2}$ Then $\tilde{H}$ is an irreducible abelian algebraic group of automorphisms in $\Omega$ (an $l$-dimensional torus). We shall now prove the

\section{Lemma. $H$ is an abelian group.}

Proof. If we recall the form of the irreducible $\Re$-modules we see that $\mathfrak{Q}$ is generated by the $f$ and a set $X$ of elements $x$ such that $\left[x e_{i}\right]=$ $0, i=1,2, \cdots, l$ and $[x h]=\Lambda(h) x, \Lambda(h) \in \Phi \quad([5]$ p. 44). We fix $i$ and write $F=a d f_{i}, E=a d e_{i}$. Then if $x_{0}=x \in X$ and we define $x_{j}=x_{0} F^{j}$, it is known that $x=x_{0}, x_{1}, \cdots, x_{m}$ are linearly independent and satisfy:

$$
\begin{array}{rll}
x_{j} F=x_{j+1}, \quad j=0, \cdots, m-1, & x_{m} F=0 \\
x_{0} E=0, \quad x_{j} E=-j(m-j+1) x_{j}, & j=1, \cdots, m .
\end{array}
$$

Hence $x_{0} \exp \xi E=x_{0}$,

$$
\begin{gathered}
x_{0} \exp \xi^{-1} F=\sum_{0}^{m} \frac{\xi^{-j}}{j !} x_{j} \\
x_{j}(\exp \xi E)=\sum_{l=0}^{j}(-1)^{l} \prod_{k-1}^{l}(m-j+k)\left(\begin{array}{l}
j \\
l
\end{array}\right) \xi^{l} x_{j-l} .
\end{gathered}
$$

Hence $x_{0}(\exp \xi E)\left(\exp \xi^{-1} F\right)(\exp \xi E)=\sum_{r=0}^{m} a_{r} \xi^{-r} x_{r}$ where

$$
\begin{aligned}
a_{r} & =\sum_{l=0}^{m-r}(-1)^{l}\left(\begin{array}{c}
r+l \\
l
\end{array}\right) \prod_{k=1}^{l}(m-r-l+k) \frac{1}{(r+l) !} \\
& =\frac{1}{r !} \sum_{l=1}^{m-r}(-1)^{l}\left(\begin{array}{c}
m-r \\
l
\end{array}\right) .
\end{aligned}
$$

It follows that $a_{r}=0$ if $r \neq m$ and $a_{m}=1 / m$ ! Hence

$$
x_{0}(\exp \xi E)\left(\exp \xi^{-1} F\right)(\exp \xi E)=(1 / m !) \xi^{-m} x_{m} .
$$

A similar calculation shows that

$$
x_{m}(\exp \xi E)\left(\exp \xi^{-1} F\right)(\exp \xi E)=(-1)^{m} m ! \xi^{m} x_{0} .
$$

\section{Hence}

${ }^{2}$ [8] p. 446. A simpler proof will be given in a forthcoming book on Lie algebras by the author. 


$$
x \omega_{i}(\xi)=\xi^{-m} x
$$

where $m$ is a nonnegative integer. This implies that $x \omega_{i}(\xi) \omega_{i^{\prime}}\left(\xi^{\prime}\right)=$ $x \omega_{i^{\prime}}\left(\xi^{\prime}\right) \omega_{i}(\xi)$ if $x \in X$. In view of $(6), \omega_{i}(\xi)$ and $\omega_{i}\left(\xi^{\prime}\right)$ commute also in their action on the $f_{j}$. It follows that the group $H$ generated by the $\omega_{i}(\xi)$ is abelian.

Now let $\rho$ be a semi-simple automorphism of the semi-simple Lie algebra $\&$ and let $\mathfrak{I}_{1}(\rho)$ be the set of $\rho$-fixed elements. We have $\mathfrak{R}_{1}(\rho)=$ $\Omega \oplus \mathbb{S}$ where $\Omega$ is the derived algebra and $\sqrt{S}$ is the center. Then $\Omega$ is semi-simple and we can apply the above considerations. Then let $\mathfrak{S}$ be a Cartan subalgebra of $\Re, e_{i}, f_{i}, h_{i}$ canonical generators of the type indicated such that the $h_{i}$ form a basis for $\mathfrak{S}$. Let $H$ be the abelian group of automorphisms of $\mathbb{2}$ generated by the $\omega_{i}(\xi), \tilde{H}$ its restriction to $\Omega$. Then if $\mu_{1}, \mu_{2}, \cdots, \mu_{l}$ are arbitrary nonzero elements of $\Phi$, the automorphism $\zeta\left(\mu_{1}, \cdots, \mu_{l}\right)$ of $\Omega$ such that $e_{i} \rightarrow \mu_{i} e_{i}, f_{i} \rightarrow \mu_{i}^{-1} f_{i}$ belongs to $\tilde{H}$. It is known that $\Omega$ has a basis consisting of the $h_{i}$, certain products $\left[\cdots\left[e_{i_{1}} e_{i_{2}}\right] \cdots e_{i_{r}}\right]$ and certain products $\left[\cdots\left[f_{i_{1}} f_{i_{2}}\right] \cdots f_{i_{r}}\right]$. The first of these is a characteristic vector of $\zeta\left(\mu_{1}, \cdots, \mu_{l}\right)$ belonging to $\mu_{i_{1}} \mu_{i_{2}} \cdots \mu_{i_{r}}$ and the second belongs to the root $\left(\mu_{i_{1}} \mu_{i_{2}} \cdots \mu_{i_{r}}\right)^{-1}$. It follows that the $\mu^{\prime}$ 's can be chosen so that $\hat{S}$ is the characteristic space of the root 1 of $\zeta\left(\mu_{1}, \cdots, \mu_{l}\right)$. It is clear that any $\zeta \in H$ commutes with $\rho$ and so it respects the decomposition: $\&=\mathfrak{L}_{1}(\rho) \oplus \mathbb{2}^{1-\rho}$, $\mathfrak{2}^{1-\rho}=$ $\left\{x-x^{\rho}\right\}$. It follows by the standard specialization argument that there exists an automorphism $\eta=\rho \zeta, \zeta \in H$, such that $\mathfrak{2}_{1}(\eta)=\mathfrak{g} \oplus \mathfrak{S}$. Since $\Omega_{1}(\eta)$ is abelian we see that $\eta$ is regular.

Let $H_{0}(\eta)$ be the algebraic component of 1 in the group of automorphisms of \& commuting with $\eta$. Since $H$ is abelian, $\omega_{i}(\xi)$ commutes with $\eta$. It follows from (8) that for fixed $i, \xi \neq 0$ in $\Phi$, the $\omega_{i}(\xi)$ form an irreducible algebraic group. Hence $\omega_{i}(\xi) \in H_{0}(\eta)$ and $H \subseteq H_{0}(\eta)$. Then $\zeta^{-1} \in H_{0}(\eta)$ and $\rho=\eta \zeta^{-1}$.

We have therefore proved

THEOREM 5. If $\rho$ is a semi-simple automorphism of a semi-simple Lie algebra then $\rho$ has the form $\eta \zeta$ where $\eta$ is regular and $\zeta \in H_{0}(\eta)$ the component of 1 of the group of automorphisms commuting with $\eta$.

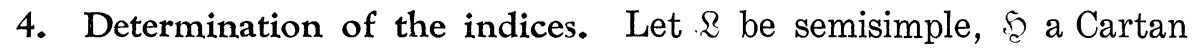
subalgebra, $e_{i}, f_{i}, h_{i}, i=1,2, \cdots, l$ canonical generators such that the $h_{i}$ form a basis for $\mathfrak{S}$ and (3) hold. We define the group of automorphisms of the Cartan matrix $\left(A_{i j}\right)$ to be the subgroup of the symmetric group $S_{l}$ on $1,2, \cdots, l$ of the permutations $s$ such that $A_{s(i), s(j)}=A_{i, j}$, $i, j=1,2, \cdots, l$. If $s$ is in this group there is a unique automorphism $\sigma$ of $\&$ such that $e_{i}^{\tau}=e_{s(i)}, f_{i}^{\sigma}=f_{s(i)}$. The set of these automorphisms is a finite group $F$ isomorphic to the group of automorphisms of the 
Cartan matrix. It is known that $G=G_{0} F=F G_{0}{ }^{3}$ '

Let $\sigma \in F, s$ the corresponding automorphism of the Cartan matrix. If $\mu_{i}, i=1,2, \cdots, l$, are arbitrary nonzero elements of $\Phi$ then there exists an automorphism $\zeta$ of $\mathfrak{Q}$ such that

$$
e_{i}^{\zeta}=\mu_{i} e_{i}, \quad f_{i}^{\zeta}=\mu_{i}^{-1} f_{i} .
$$

The argument used in proving Theorem 5 shows that $\zeta \in G_{0}$. We choose $\mu_{i}$ so that

$$
\mu_{s(i)}=\mu_{i}
$$

for every $i$, and for every positive root $\alpha=\Sigma \kappa_{i} \alpha_{i}, \kappa_{i}$ nonnegative integral,

$$
\left(\mu_{1}^{k_{1}} \mu_{2}^{\kappa_{2}} \cdots \mu_{l}^{\kappa_{l}}\right)^{m} \neq 1 \text {, }
$$

$m$ the order of $s$. Clearly such a choice of the $\mu_{i}$ can be made. Also it is evident that $\zeta$ is semi-simple and $\mathscr{L}_{1}\left(\zeta^{m}\right)=\mathfrak{g}$. We have $\sigma \zeta=\zeta \sigma$ and $\sigma^{m}=1$ so that $\sigma$ is semi-simple. Hence $\eta=\sigma \zeta$ is semi-simple and $\mathfrak{Z}_{1}(\eta)$ is the set of $\eta$-fixed points. If $x \in \mathfrak{L}_{1}(\eta), x^{\eta^{m}}=x^{\zeta^{m}}=x$ so $x \in \mathfrak{S}$. Since $x^{\zeta}=x$ for $x \in \mathfrak{S}$ we have $x^{\sigma}=x^{\zeta \sigma}=x^{\eta}=x$. Conversely, if $x \in \mathfrak{S}$ and $x^{\sigma}=x$ then $x \in \mathscr{Z}_{1}(\eta)$. Hence $\mathfrak{Z}_{1}(\eta)$ is the subspace of $\mathfrak{Q}$ of $\sigma$-fixed elements. We have $h_{i}^{\sigma}=h_{s(i)}$ for the basis $\left(h_{1}, h_{2}, \cdots, h_{l}\right)$ of $s_{i}$. Let

$$
s=\left(i_{1} \cdots i_{m_{1}}\right)\left(j_{1} \cdots j_{m_{2}}\right) \cdots\left(u_{1} \cdots u_{m_{p}}\right)
$$

be the decomposition of the permutation $s$ into disjoint cycles of length $\geqq 1\left(m_{1}+m_{2}+\cdots+m_{p}=l\right)$. Then it is clear that the elements

$$
g_{1}=h_{i_{1}}+\cdots+h_{i_{m_{1}}}, \cdots, g_{p}=h_{u_{1}}+\cdots+h_{u_{m_{p}}}
$$

constitute a basis for the subspace of $\mathfrak{S}$ of $\sigma$-fixed points. Hence $\operatorname{dim} \Re_{1}(\eta)=p$, the number of cycles in the decomposition of $s$. Since $\zeta \in G_{0}, \eta$ and $\sigma$ are in the same component of the automorphism group. Since $\mathfrak{R}_{1}(\eta) \subseteq \mathfrak{S}, \mathbb{R}_{1}(\eta)$ is abelian and so $\eta$ is a regular element in the same component as $\sigma$. We can therefore state

THEOREM 6. Let $G_{i}=\sigma_{i} G_{0}$ be a component of the group of automorphisms where $\sigma_{i} \in F$ and corresponds to the automorphism $s_{i}$ of the Cartan matrix. Then the index of $G_{i}$ is the number of cycles in the decomposition of $s_{i}$ into disjoint cycles of lengths $\geqq 1$.

For $G_{0}$ we may take $\sigma_{0}=1$ and we obtain that the index of $G_{0}$ is $l$, the dimensionality of the Cartan subalgebra $\mathfrak{g}$. On the other hand. it is clear that if $\sigma_{i} \neq 1$ then the index of $\sigma_{i} G_{0}$ is positive but is less than $l$. Hence $\sigma_{i} G_{0} \neq G_{0}$ and the decomposition $G=F G_{0}$ is semidirect.

${ }^{3}$ The arguments of [3] can be used to prove this. A detailed discussion will be given in Chapter IX of the author's forthcoming book. 
Corollary. The index of $G_{0}$ is $l$ and the index of any $G_{i} \neq G_{0}$ is positive and less than $l$. The decomposition $G=F G_{0}$ is semi-direct.

Our analysis shows also that any Cartan subalgebra is the space $\mathfrak{R}_{1}(\eta)$ for a regular automorphism belonging to $G_{0}$. Hence Theorem 4 proves again the conjugacy theorem for Cartain subalgebras by means of an element of $G_{0}$. Theorem 4 can be considered as a natural generlization of the classical conjugacy theorem.

Let $\eta=\sigma \zeta$ be the automorphism which we constructed for the proof of Theorem 6. If $\nu_{1}, \nu_{2}, \cdots, \nu_{l}$ are nonzero elements of $\Phi$ such that $\nu_{s(i)}=$ $\nu_{i}, i=1,2, \cdots, l$, then it is clear that the automorphism $\zeta\left(\nu_{1}, \nu_{2}, \cdots, \nu_{l}\right)$ such that

$$
e_{i}^{\zeta\left(\nu_{1}, \cdots, \nu_{l}\right)}=\nu_{i} e_{i}, \quad f_{i}^{\zeta\left(\nu_{1}, \cdots, \nu_{1}\right)}=\nu_{i}^{-1} f_{i}
$$

commutes with $\eta$. The set of these automorphisms is an irreducible algebraic group of $p$ dimensions where $p$ is the index of $\eta G_{0}$. It follows that this group coincides with $H_{0}(\eta)$ the component of 1 in the group of automorphisms commuting with $\eta$. Theorem 4 therefore implies that if $\rho$ is any semi-simple automorphism then $\rho$ has the form $\tau\left(\sigma_{i} \xi\right) \tau^{-1}$ where $\tau \in G_{0}, \sigma_{i} \in F$ and $\xi$ is of the form $\zeta\left(\nu_{1}, \cdots, \nu_{l}\right)$ as in (14). This is Gantmacher's "canonical form" for the semi-simple automorphism $\rho$. It is clear from the definition of $\sigma_{i}$ that $\mathfrak{S}^{\sigma_{i}} \leqq \mathfrak{S}_{\text {. }}$. In fact, if we choose the basis for $\mathfrak{S}_{2}$ as before, then $h_{j}^{\sigma}{ }^{\sigma}=h_{s_{i}(j)}$ where $s_{i}$ is the permutation of $1,2, \cdots, l$ associated with $\sigma_{i}$. It is clear that the restriction of $\sigma_{i}$ to $\mathfrak{S}$ is periodic and the subspace of $\sigma_{i}$-fixed points of $\mathfrak{S}$ has $l_{i}$-dimensions where $l_{i}$ is the index of the component $G_{0}^{r}$. Since $h^{\xi}=h$ for every $h \in \mathfrak{S}$ these results hold also for $\sigma_{i} \xi$. Since $\rho=\tau\left(\sigma_{i} \xi\right) \tau^{-1}$ we have the following

THEOREM 7. If $\rho$ is a semi-simple automorphism of a semi-simple Lie algebra, then there exists a Cartan subalgebra $\mathfrak{S}$ such that $\mathfrak{S}^{\circ} \subseteq \mathfrak{W}$, the restriction of $\rho$ to $\mathfrak{S}$ is periodic and the subspace of $\rho$-fixed points of $\mathfrak{S}$ has dimensionality equal to the index of the component $\rho G_{0}$.

We look next at the indices of the components $G_{i} \neq G_{0}$ and for the sake of simplicity we confine our attention to the simple algebras. Outer automorphisms (that is, automorphisms not in $G_{0}$ ) exist in the following cases: $A_{l}, l>1, D_{l}, l \geqq 4$ and $E_{6}$ and only in these cases, The group of automorphisms of the Cartan matrix can be identified with the group of automorphisms of the associated Dynkin diagram. For $A_{l}$ the automorphism $\neq 1$ of the Dynkin diagram (suitably labelled) is $i \rightarrow l+1-i$. If $l$ is even the cycle decomposition is $(1 l)(2, l-1) \cdots(l / 2, l / 2+1)$ and for odd $l$ it is $(1 l)(2, l-1) \cdots((l-1) / 2,(l-1) / 2+2)((l-1) / 2+1)$. In 
both cases, if $G_{1} \neq G_{0}$, then index $G_{1}=[(l+1) / 2]$. For $D_{l}, l>4, G=$ $G_{0} \cup G_{1}, G_{1} \neq G_{0}$ and the permutation associated with $G_{1}$ is $i \rightarrow i$ if $i \leqq l-2, l \rightarrow l-1, l-1 \rightarrow l$. The cycle decomposition is (1) $\cdots(l-2)$ $(l-1, l)$. Hence index $G_{1}=l-1$. For $D_{4}$ the group of automorphisms of the Dynkin diagram is the symmetric group on $1,3,4$ if $\alpha_{1}, \alpha_{3}, \alpha_{4}$ are the end points of the diagram. If the permutation associated with $G_{i}$ is of order two then index $G_{i}=3$. If the permutation is of order three then index $G_{i}=2$. For a suitable ordering of the vertices the automorphism $\neq 1$ of the Dynkin diagram of $E_{6}$ is (15) (24) (3) (6). Hence index $G_{1}=4$.

Theorem 8. For $A_{l}, l>1, G=G_{0} \cup G_{1}$ and index $G_{1}=[(l+1) / 2]$. For $D_{l}, l>4, G=G_{0} \cup G_{1}$ and index $G_{1}=l-1$. For $D_{4}, G / G_{0}$ is the symmetric group $S_{3}$ and index $G_{i}=2$ if the coset of $G_{i}$ is of order 3 and index $G_{i}=3$ if the coset of $G_{i}$ is of order 2 . For $E_{6}, G=G_{0} \cup G_{1}$ and index $G_{1}=4$.

5. Application to fixed points. In the applications to fixed points we can for the most part relax the assumption on the base field $\Phi$ and suppose only that $\Phi$ is of characteristic 0 . If $\eta$ is an automorphism in $\&$ over $\Phi$ and $P$ is an extension field of $\Phi$ then $\eta$ has a unique extension to an automorphism $\eta$ of $\mathfrak{R}_{P}$ and the space of $\eta$-fixed points of $\mathfrak{L}_{P}$ has the form $\widetilde{\mho}(\eta)_{P}$ where $\widetilde{\mho}(\eta)$ is the space of $\eta$-fixed points of \&. This remark reduces most considerations of fixed points to the algebraically closed case.

The following result is due to Borel and Mostow ([1] p 398) for semi-simple automorphisms.

THEOREM 9. If \& is a nonsolvable Lie algebra over a field of characteristic 0 then any automorphism $\eta$ of $\&$ has a fixed point.

Proof. It suffices to assume the base field is algebraically closed. Let $\Re$ be the radical of $\mathcal{L}$. Then $\eta$ induces an automorphism $\bar{\eta}$ in the semi-simple Lie algebra $\bar{\Sigma}=2 / \Re$. By the Corollary to Th. $6, \operatorname{dim} \overline{\mathbb{S}}_{1}(\bar{\eta}) \geqq 1$. This means that 1 is a characteristic root of $\bar{\eta}$. Hence 1 is a characteristic root of $\eta$ and there exists a nonzero fixed point.

It is convenient at this point to introduce another type of index $m\left(G_{i}\right)$ of a component $G_{i}$ of the automorphism group in the algebraically closed case. We set $m\left(G_{i}\right)=$ minimum of $\operatorname{dim} \mathfrak{F}(\eta)$ for $\eta \in G_{i}$ where $\mathfrak{F}(\eta)$ is the space of $\eta$-fixed points. If $(\alpha)$ is a matrix of $\eta$ then $\operatorname{dim} \mathfrak{\xi}(\eta)=$ $n-\operatorname{rank}((\alpha)-1), n=\operatorname{dim} 2$. Hence $m\left(G_{i}\right)=k_{i}$ means that for every $\eta \in G_{i}$ every minor of order $n-k_{i}+1$ of $(\alpha)-1$ vanishes but there exists an $\eta \in G_{i}$ such that $(\alpha)-1$ has a nonvanishing minor of order $n-k_{i}$. If is clear from this that the elements $\eta$ of $G_{i}$ such that 
$\operatorname{dim} \mathfrak{F}(\eta)=k_{i} \equiv m\left(G_{i}\right)$ form an open set in $G_{i}$. Hence this set contains a regular element $\eta$. If $\mathscr{Q}$ is semisimple it follows that such an $\eta$ is semi-simple. Then $\mathfrak{F}(\eta)=\mathfrak{R}_{1}(\eta)$ and consequently $m\left(G_{i}\right)=n\left(G_{i}\right)$. We can state this result in the following way.

THEOREM 10. Let 2 be a semi-simple Lie algebra over an algebraically closed field of characteristic 0 and let $l_{i}$ be the index of the component $G_{i}$ of the group of automorphisms of $\mathrm{L}$. Let $\eta \in G_{i}$ and let $i(\eta)$ be the space of fixed points under $\eta$. Then $\operatorname{dim} \mathfrak{F}(\eta) \geqq l_{i}$ and there exists $\eta \in G_{i}$ such that $\operatorname{dim} \mathfrak{F}(\eta)=l_{i}$.

This result can also be applied to the case of an arbitrary base field of charactetistic 0 . A given automorphism $\eta$ has its extension to an automorphism $\eta$ of $\Omega_{\Omega}, \Omega$ the algebraic closure of $\Phi$. Also $\Omega_{\Omega}$ is semisimple if $\Omega_{\Omega}$ is semi-simple. The result just proved gives a lower bound for $\operatorname{dim} \mathfrak{F}(\eta)$ once the component of $\eta$ in the group of automorphisms of $\Omega_{\Omega}$ is known. Even without this information we can say that $\operatorname{dim} \mathfrak{F}(\eta) \geqq m$ where $m$ is the minimum of the indices of the components $G_{i}$ of the group of automorphisms of $\mathfrak{R}_{\Omega}$.

Again let $\&$ be semi-simple over any field of characteristic 0 and let $\eta$ be semi-simple in the sense that its minimum polymonial is a product of distinct prime factors. Since the base field is perfect this property is preserved under field extension. It follows from this that $\Omega_{1}(\eta)=\Im(\eta)$ is reductive. This implies that any Cartan subalgebra $\mathfrak{L}_{1}$ of $\mathscr{R}_{1}(\eta)$ is abelian and reductive in $\mathbb{2}$. Moreover, any reductive abelian subalgebra of $\mathscr{R}_{1}(\eta)$ can be imbedded in a Cartan subalgebra and any two Cartan subalgebras of $\mathfrak{R}_{1}(\eta)$ have the same dimensionality. It follows by a field extension argument and Th. 7 that the dimensionality of $\mathfrak{F}_{1}$ is not less than the index of the component of $\eta$ in the group of automorphisms of $\Omega_{\Omega}, \Omega$ the algebraic closure of the base field.

The result just indicated holds also for arbitrary 2 and semi-simple $\eta$ by virtue of a result of Mostow's that there exists a Levi-decomposition $\mathbb{R}=\Re+\Re$ where $\Re$ is the radical and $\Re$ is a semi-simple subalgebra invariant under $\eta$ ([7]). It is known that if $a \in \Re$ and $a d_{\Re} a$ is semisimple then $a d_{\varsigma} a$ is semi-simple. We can therefore state the following extension of a theorem of de Siebenthal-Borel-Mostow ([1] p. 498).

THEOREM 11. Let $\eta$ be a semi-simple automorphism of a Lie algebra $\mathfrak{Q}$ over a field of characteristic 0 . Let $\Re$ be the radical, $\overline{\mathbb{Z}}=\mathfrak{Q} / \mathfrak{R}$ and let $m$ be the minimal index of the components of the group of automorphism of $\overline{\mathfrak{S}}_{\Omega}, \Omega$ the algebraic closure of the base field. Then $m \geqq 1$ and there exists an abelian reductive subalgebra of $m$ dimensions whose elements are fixed under $\eta$. 


\section{BIBLIOGRAPHY}

1. A. Borel and G. D. Mostow, On semi-simple automorphisms of Lie algebras, Annals of Math., 61 (1955), 389-405.

2. H. Cartan and C. Chevalley, Sśminaire École Normale Supérieure, Gáomátrie Algábrique, 1956.

3. C. Chevalley, Thíorie des Groupes de Lie, Tome II (1951) and Tome III (1955).

4. F. Gantmacher, Canonical representation of automorphisms of a complex semi-simple Lie algebra, Mat. Sbornik 5(47) (1939), 101-146.

5. Harish-Chandra, On some applications of the universal enveloping algebra of a semisimple Lie algebra, Trans. Amer. Math. Soc., 70 (1951), 28-96.

6. N. Jacobson, Completely reducible Lie algebras of linear transformations, Proc. Amer. Math. Soc., 2 (1951), 105-113.

7. G. D. Mostow, Fully reducible subgroups of algebraic groups, Amer. J. Math., 78 (1956), 200-221.

8. G. B. Seligman, On automorphisms of Lie algebras of classical type, Trans. Amer. Math. Soc., 92 (1959), 430-448.

YALE UNIVERSITY 



\section{PACIFIC JOURNAL OF MATHEMATICS}

\section{EDITORS}

Ralph S. Phillips

Stanford University

Stanford, California

M. G. Arsove

University of Washington

Seattle 5, Washington
A. L. Whiteman

University of Southern Californla

Los Angeles 7, California

Lowell J. Paige

University of California

Los Angeles 24, California

\section{ASSOCIATE EDITORS}

E. F. BECKENBACH

D. DERRY

H. L. ROYDEN

E. G. STRAUS

T. M. CHERRY

M. OHTSUKA

E. SPANIER

F. WOLF

\section{SUPPORTING INSTITUTIONS}

UNIVERSITY OF BRITISH COLUMBIA

STANFORD UNIVERSITY

CALIFORNIA INSTITUTE OF TECHNOLOGY

UNIVERSITY OF CALIFORNIA

MONTANA STATE UNIVERSITY

UNIVERSITY OF TOKYO

UNIVERSITY OF UTAH

UNIVERSITY OF NEVADA

NEW MEXICO STATE UNIVERSITY

OREGON STATE UNIVERSITY

UNIVERSITY OF OREGON

OSAKA UNIVERSITY

WASHINGTON STATE UNIVERSITY

UNIVERSITY OF WASHINGTON

UNIVERSITY OF SOUTHERN CALIFORNIA

AMERICAN MATHEMATICAL SOCIETY CALIFORNIA RESEARCH CORPORATION SPACE TECHNOLOGY LABORATORIES NAVAL ORDNANCE TEST STATION 


\section{Pacific Journal of Mathematics}

\section{Vol. 12, No. $1 \quad$ January, 1962}

Jonathan L. Alperin, Groups with finitely many automorphisms $\ldots \ldots \ldots \ldots \ldots \ldots \ldots \ldots$

Martin Arthur Arkowitz, The generalized Whitehead product ................ 7

John D. Baum, Instability and asymptoticity in toplogical dynamics . . . . . . . . . . 25

William Aaron Beyer, Hausdorff dimension of level sets of some Rademacher series .... $\quad 35$

Frank Herbert Brownell, III, A note on Cook's wave-matrix theorem . . . . . . . . . . . . . 47

Gulbank D. Chakerian, An inequality for closed space curves ................. 53

Inge Futtrup Christensen, Some further extensions of a theorem of Marcinkiewicz ....... 59

Charles Vernon Coffman, Linear differential equations on cones in Banach spaces . . . . . 69

Eckford Cohen, Arithmetical notes. III. Certain equally distributed sets of integers . . . . . 77

John Irving Derr and Angus E. Taylor, Operators of meromorphic type with multiple poles

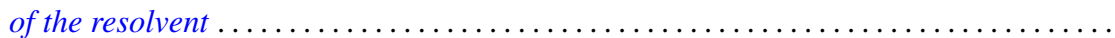

Jacob Feldman, On measurability of stochastic processes in products space .............

Robert S. Freeman, Closed extensions of the Laplace operator determined by a general class of boundary conditions, for unbounded regions ......................

Robert E. Fullerton, Geometric structure of absolute basis systems in a linear topological

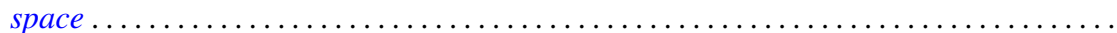

Dieter Gaier, On conformal mapping of nearly circular regions

Andrew Mattei Gleason and Hassler Whitney, The extension of linear functionals defined

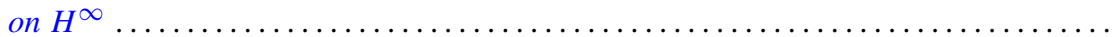

Seymour Goldberg, Closed linear operators and associated continuous linear

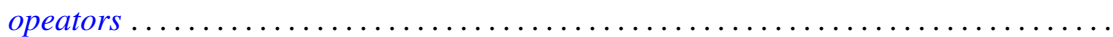

Basil Gordon, Aviezri Siegmund Fraenkel and Ernst Gabor Straus, On the determination of sets by the sets of sums of a certain order

Branko Grünbaum, The dimension of intersections of convex sets. .

Paul Daniel Hill, On the number of pure subgroups

Robert Peter Holten, Generalized Goursat problem . .

Alfred Horn, Eigenvalues of sums of Hermitian matrices ...........

Henry C. Howard, Oscillation and nonoscillation criteria for

$$
y^{\prime \prime}(x)+f(y(x)) p(x)=0
$$

Taqdir Husain, $S$-spaces and the open mapping theorem ...

Richard Eugene Isaac, Markov processes and unique stationary probability measures ...

John Rolfe Isbell, Supercomplete spaces ....................

John Rolfe Isbell, On finite-dimensional uniform spaces. II .........

N. Jacobson, A note on automorphisms of Lie algebras ..............

Antoni A. Kosinski, A theorem on families of acyclic sets and its applications

Marvin David Marcus and H. Minc, The invariance of symmetric functions of singular values...

Ralph David McWilliams, A note on weak sequential convergence.

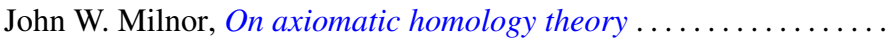

Victor Julius Mizel and Malempati Madhusudana Rao, Nonsymmetric projections in

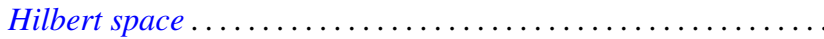

Calvin Cooper Moore, On the Frobenius reciprocity theorem for locally compact

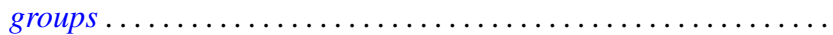

Donald J. Newman, The Gibbs phenomenon for Hausdorff means . 